\title{
Wie wird der Innovationsfonds
} innovativ?

VOLKER ULRICH, CHRISTIAN MAIER, ANNA-LENA TRESCHER

Prof. Dr. Volker Ulrich ist Inhaber des Lehrstuhls für Volkswirtschaftslehre III, insb. Finanzwissenschaft an der Universität Bayreuth, Mitglied in zahlreichen Ausschüssen zum Gesundheitswesen und der Ökonomie, unter anderem ist er Mitglied im Wissenschaftlichen Beirat des Bundesversicherungsamts und im Erweiterten Vorstand der Deutschen Gesellschaft für Gesundheitsökonomie

Dr. Christian Maier ist akademischer Rat am Lehrstuhl für Volkswirtschaftslehre III an der Universität Bayreuth mit Schwerpunkten auf den Themen Gesundheitsökonomie, Pflegeversicherung und Nachhaltigkeit

M.Sc. Anna-Lena Trescher ist wissenschaftliche Mitarbeiterin am Lehrstuhl für Volkswirtschaftslehre III an der Universität Bayreuth mit einem Schwerpunkt auf dem Thema Gesundheitsökonomie

Das deutsche Gesundheitswesen ist nach wie vor stark sektoral geprägt. Das gilt auch für den Gemeinsamen Bundesausschuss (G-BA), das höchste Entscheidungsgremium der Selbstverwaltung. Entscheidungen werden dort vor allem mit Blick auf die jeweiligen Sektorinteressen getroffen. Auf dieses System trifft nun durch den neuen Fonds ein finanzieller Impuls, der sektorunabhängig Prozessinnovationen anstoßen soll. Kann das überhaupt funktionieren? In dem folgenden Beitrag argumentieren wir, dass der Innovationsfonds eine starke Orientierung an der Nachfrageseite erhalten und eine Förderung von Prozessinnovationen auf der Grundlage der besonderen Versorgungsformen vornehmen sollte. Die konkrete Umsetzung wird entscheiden, ob und wie effizient, effektiv und nachhaltig der Innovationsfonds sein wird oder ob er sich doch wieder in der Befriedigung sektoraler Ansprüche verliert.

\section{Innovationsförderung im Gesundheitswesen}

Der Innovationsfonds kommt - und mit ihm die Frage, wie durch seine bestimmungsgerechte Ausgestaltung ein sinnvoller und verantwortungsbewusster Einsatz der veranschlagten Mittel sichergestellt werden kann, mit dem Ziel, das GKV-System durch innovative Lösungen an der Schnittstelle zwischen ambulanter und stationärer Versorgung weiterzuentwickeln. Darüber hinaus gilt es zu klären, wie der Innovationsfonds als neues Instrument vor dem Hintergrund der bisherigen Erfahrungen im Bereich der Integrierten Versorgung in Deutschland aufgestellt werden muss, um eine nachhaltige Förderung und Entwicklung sektorübergreifender Versorgungselemente und -prozesse zu ermöglichen.
Im Status quo besteht nur eine geringe Innovationsbereitschaft der Akteure, bedingt durch die Fesseln des Kollektivvertrags und die Finanzarchitektur des Gesundheitsfonds. Innerhalb dieses hoch regulierten Betätigungsumfeldes entfalten sich innovative Ansätze nur innerhalb enger Grenzen. Dies spiegelt sich auch in den beobachtbaren Rent-Seeking-Aktivitäten der Krankenkassen wieder (u.a. auf Grund nicht unmittelbar internalisierbarer Gewinne), so dass dem medizinisch-technischen Fortschritt nahezu ausschließlich anhand von Produktinnovationen Rechnung getragen wird. Folglich bedarf es bei der Ausgestaltung des Innovationsfonds konkreter Regelungen und eines festen Kriterienkatalogs, damit dieser seiner Bestimmung auch nachkommen kann: die Förderung sektorübergreifender bzw. noch stärker, sektorunab- 
hängiger, Prozessinnovationen, die nach der subventionierten Einführungsphase auch Chancen aufweisen, sich am Markt selbstständig zu behaupten.

Ökonomische Überlegungen zur Wettbewerbsintensität basieren auf der Erkenntnis, dass Marktunvollkommenheiten mit Blick auf technischen Fortschritt sogar erwünscht sein können, da nur bei Abweichungen von der Nullgewinnannahme ein Anreiz für Innovationen besteht (vgl. Kantzenbach 1966). Allerdings müssen die Strukturen dazu geeignet sein. Ein funktionsfähiger Wettbewerb in der GKV, bei starken sektoralen Interessen der Leistungserbringer, setzt eine Stärkung der Nach-

\section{Ein funktionsfähiger Wettbewerb setzt eine Stärkung der Nachfrageseite voraus: Krankenkassen und Versicherte/ Patienten sollten daher Gestaltungskompetenz beim Innovationsfonds bekommen.}

unter perfektem Wettbewerb, zu agieren. Dieses Marktversagen wird insbesondere von Informationsasymmetrien, externen Effekten, Risiko- und Selbstselektion sowie durch Moral-Hazard-Verhalten ausgelöst.

Grundsätzlich unterscheidet sich der Gesundheitsmarkt aufgrund seiner erwünschten Sozialfunktion sowie dem Austausch von Erfahrungs- und Vertrauensgütern signifikant von anderen Märkten für Produkte und Dienstleistungen. Obwohl dem Marktansatz in der Gesundheitsversorgung aufgrund der genannten Spezifika Grenzen gesetzt sind, sollten wichtige Funktionen des Wettbewerbs auch im Gesundheitswesen gelten: So kommt neben der Allokations-, Anreiz- und Kontrollfunktion insbesondere der Innovationsfunktion des Wettbewerbs eine besondere Bedeutung zu. Hierbei ermöglicht Wettbewerb die Entwicklung von Produkt- und Prozessinnovationen im Zuge dezentraler Suchprozesse. Im Sinne Hayeks charakterisiert sich Wettbewerb somit als Such- und Entdeckungsverfahren mit evolutorischer Problem-

frageseite voraus, bestehend aus Krankenkassen und Versicherten/Patienten. Ihnen sollte daher Gestaltungskompetenz beim Innovationsfonds zukommen, beispielsweise bereits bei der Kriteriensetzung.

Dadurch ließe sich auch die nachhaltige Verwendung von Versichertengeldern stärken, da Projekte, nach positiver Evaluation, nicht länger bestimmten Sektoren zugeschlagen werden. Die sektorale Zuordnung widerspricht der Idee des Innovationsfonds und dürfte letztlich zur Verschwendung von Versichertengeldern in nicht unerheblicher Höhe beitragen. Im Folgenden gilt es zu klären, wie das in diesem Bereich verhältnismäßig starre „GKV-System“ den Impuls durch den Innovationsfonds verarbeiten und möglichst zielkonform umsetzen kann.

\section{2. Ökonomischer Rahmen für Innovationen}

Der ökonomische Nobelpreisträger Kenneth Arrow wies in seinem berühmten Aufsatz „Uncertainty and the Welfare Economics of Medical Care" bereits im Jahr 1963 darauf hin, dass die Eigenschaften von Angebot und Nachfrage im Gesundheitswesen die Gesundheitsmärkte davon abhalten, effizient und fair, d.h. lösungsfreiheit (vgl. Hayek 1972). Die Etablierung unterschiedlichster Alternativen in der Praxis sowie deren Erprobung und Verwerfung ermöglicht die Etablierung eines selbstlernenden Systems. In Bezug auf interessiert in der ökonomischen Theorie vor allem die dynamische Effizienz. Diese steht für eine optimale Innovationsrate in der Längsschnittbetrachtung und wird in den meisten Fällen sogar zu Lasten der statischen Effizienz gehen, da die meistens gewährten, zeitlich befristeten Monopole zur Refinanzierung der F\&E-Aufwendungen der statischen Effizienz entgegenstehen (etwa durch einen Patentschutz).

Um den Defiziten des Marktansatzes, welche sich für das Gesundheitswesen ergeben, entgegenzutreten und gleichzeitig aber wichtige Wettbewerbsfunktionen nutzen zu können, entwickelte Enthoven das Konzept des Managed Competition (Enthoven 1978, 1993). Dieses Konzept bietet den ordnungsökonomischen Rahmen für ein wettbewerbsorientiertes Gesundheitssystem unter Berücksichtigung der inhärenten Besonderheiten ${ }^{1}$. Nach Enthovens Argumentation haben Marktteilnehmer unter den Bedingungen des Managed Competition den Anreiz sich so zu verhalten, als befänden sie sich in die Innovationsfunktion des Wettbewerbs einem wettbewerblichen System. Damit zeigt Enthovens Modell einen Ausweg aus den Marktdefiziten auf und kann dazu beitragen, mögliche Ineffizienzen $\mathrm{zu}$ verringern oder sogar zu beseitigen. In jüngerer Zeit wurden weitere Reformkonzepte entwickelt, die sich um den Begriff „Consumer-Directed Health Care“ drehen (vgl. Buchmueller 2014, S. 820).

Wasem nennt in diesem Zusammenhang zehn Voraussetzungen für die erfolgreiche Umsetzung eines funktionsfähigen Wettbewerbs in der GKV (vgl. Wasem 2011):

1. Freie Wahl der Krankenkasse durch die Versicherten,

2. Information und Markttransparenz für die Konsumenten,

3. Preis- und Kostensensitivität von Anbietern und Nachfragern nach Versicherungsschutz und Gesundheitsleistungen,

4. Bestreitbare Versicherungs- und Gesundheitsmärkte, ${ }^{2}$

5. Vertragsfreiheit für einzelne Kassen und Leistungserbringer,

6. Effektive Wettbewerbsregulierung bezüglich Krankenversicherern und Leistungserbringern,

7. Risiko- und Einkommenssolidarität ohne Anreize zur Risikoselektion,

8. Keine Anreize für Versicherte/Patienten zum Trittbrettfahren,

9. Effektive Qualitätssicherungspolitik, 10. Garantierter Zugang zur Gesundheitsversorgung.

Mit Blick auf die angestrebte sektorunabhängige Versorgung, die der Innovationsfonds anstoßen soll, kommt den Punkten 1, 2, 5, 9 und 10 eine besondere Bedeutung zu. Die Punkte 1 und 2 betonen die Rolle nachfrageseitiger Entscheidungen im Gesundheitswesen und hier vor allem die des häufig vergessenen Akteurs „Versicherter/ Patient". Zählt man die Krankenkasse ebenfalls zu den Nachfragern/Einkäufern medizinischer Leistungen oder zumindest als Sachwalter des Patienten, kommt auch dem 5. Punkt eine wichtige wettbewerbliche Funktion zu. Der Rahmen für die Ausgestaltung der zu fördernden Projekte sollte daher auf jeden Fall selektivvertrag-

1 Hierfür hat sich in Deutschland der Begriff der Solidarischen Wettbewerbsordnung eingebürgert (vgl. Rebscher 1993, S. 4ff.).

2 Ist ein Markteintritt leicht möglich, d. h. liegen nur wenige Marktschranken vor, so spricht man von einem bestreitbaren Markt. 
lich ausgestaltet werden und eine versorgungsvertragliche Basis besitzen.

Die Möglichkeit zur Gestaltung des Versorgungsangebots ermöglicht es den Krankenkassen, sich im Wettbewerb um Versicherte zu differenzieren. Den Krankenkassen wird es ermöglicht, mit verschiedenen Leistungserbringern Selektivverträge über sektorübergreifende Prozessinnovationen zu schließen. Dies wiederum überträgt den Wettbewerb auch auf die Leistungserbringer, denn diese konkurrieren nun untereinander um die qualitativ hochwertigen Verträge mit den einzelnen Krankenkassen.

Voraussetzung für eine solche Qualitätsfunktion des Wettbewerbs bei Krankenkassen und Leistungserbringern ist, dass eine entsprechende Zielsetzung der Nachfrager, also der Krankenkassen, besteht. Nur wenn die Krankenkasse selbst ein Interesse an einer sektorübergreifenden medizinischen Versorgung ihrer Versicherten besitzt und dies bei der Vertragsgestaltung berücksichtigt, ist dies möglich. Demgegenüber verfolgen die Mandatsträger der Sektoren im G-BA überwiegend ihre jeweiligen Sektoreninteressen. Entscheidungen zur Überwindung von Sektorengrenzen gehören nicht zu ihren Aufgaben und widersprechen ihren ökonomischen Interessen. Über die Rolle des G-BA, die Förderkriterien oder einen möglichen wissenschaftlichen Beirat sowie die konkrete Auswahlentscheidung sollte daher sehr genau nachgedacht werden. Das Ziel einer wettbewerblichen Gesundheitspolitik sollte sein, entlang der Behandlungspfade Systembrüche zu vermeiden und patientenorientierte Entscheidungen nicht durch falsche ökonomische Anreize zu beeinträchtigen.

Krankenkassen- und Leistungswettbewerb sind aus dieser Sicht zwei Seiten einer Medaille. Die Möglichkeit zur Effizienzverbesserung in der Gesundheitsversorgung ergibt sich somit erst, wenn der Wettbewerb um Versicherte auf den Bereich übergreift, auf dem die tatsächlich nutzenstiftende gesundheitliche Leistungserstellung stattfindet (vgl. Wille 2009). In Bezug auf eine bessere sektorübergreifende Versorgung würde ein Übergreifen des Wettbewerbs auf die Seite der Leistungserbringer Anreize für einen Zusammenschluss von sektorenübergreifenden Leistungserbringergruppen setzen, welche über den Abbau von sektoralen Hürden innerhalb der Gruppe eine höhere Effizienz des Leistungserbringungsprozesses erzeugen können.

Insgesamt lässt sich konstatieren, dass Wettbewerb auf der Ebene der Leistungserbringung eher noch die Ausnahme darstellt. Es bestehen insgesamt nur sehr geringe Anreize für die Leistungserbringer, sich in einen Wettbewerb um selektive Verträge jenseits der kollektivvertraglichen Vereinbarungen zu begeben. Bezüglich der Innovationsfreundlichkeit des Systems bedeutet dies, dass Anreize zur Vielmehr erscheint es notwendig, die Innovationsfähigkeit des Systems weiter zu stärken. Hierzu müssen die Blockaden der Systemteilnehmer gelöst werden, indem Einschränkungen der Vertragsfreiheit abgebaut und die selektivvertraglichen Optionen ausgeweitet werden. Darüber hinaus muss seitens der Politik auch das ursächliche Problem der starken sektoralen Trennung der Leistungserbringung angegangen werden. Schließlich kann es nicht Ziel einer rationalen und ordnungspolitisch fundierten Gesundheitspolitik sein, die Sektoren über unterschiedliche Strukturbestimmungen gegeneinander abzugrenzen und somit die sektoralen Hürden weiter auszubauen, aber gleichzeitig über bestimmte Töpfe eine sektorübergreifende Versorgung zu fördern. Ohne einen gleichzeitig einhergehenden konsequenten Abbau der sektoralen Grenzen wird der Anschub innovativer sektorübergreifender Versorgungsformen kaum sein Ziel erreichen können. Vielmehr besteht die Gefahr, dass die bereitgestellten Mittel im System sektoraler Interessen versickern.

\section{Zur optimalen Ausgestaltung
des Innovationsfonds}

\subsection{Förderaspekte}

Der neue Innovationsfonds stellt zunächst einen finanziellen Impuls dar, der auf ein nach wie vor stark sektoral ausgerichtetes Gesundheitswesen trifft, dort aber Sektorübergreifendes bewirken soll. Wie kann das funktionieren bzw. wie sieht eine optimale Ausgestaltung des Innovationsfonds 


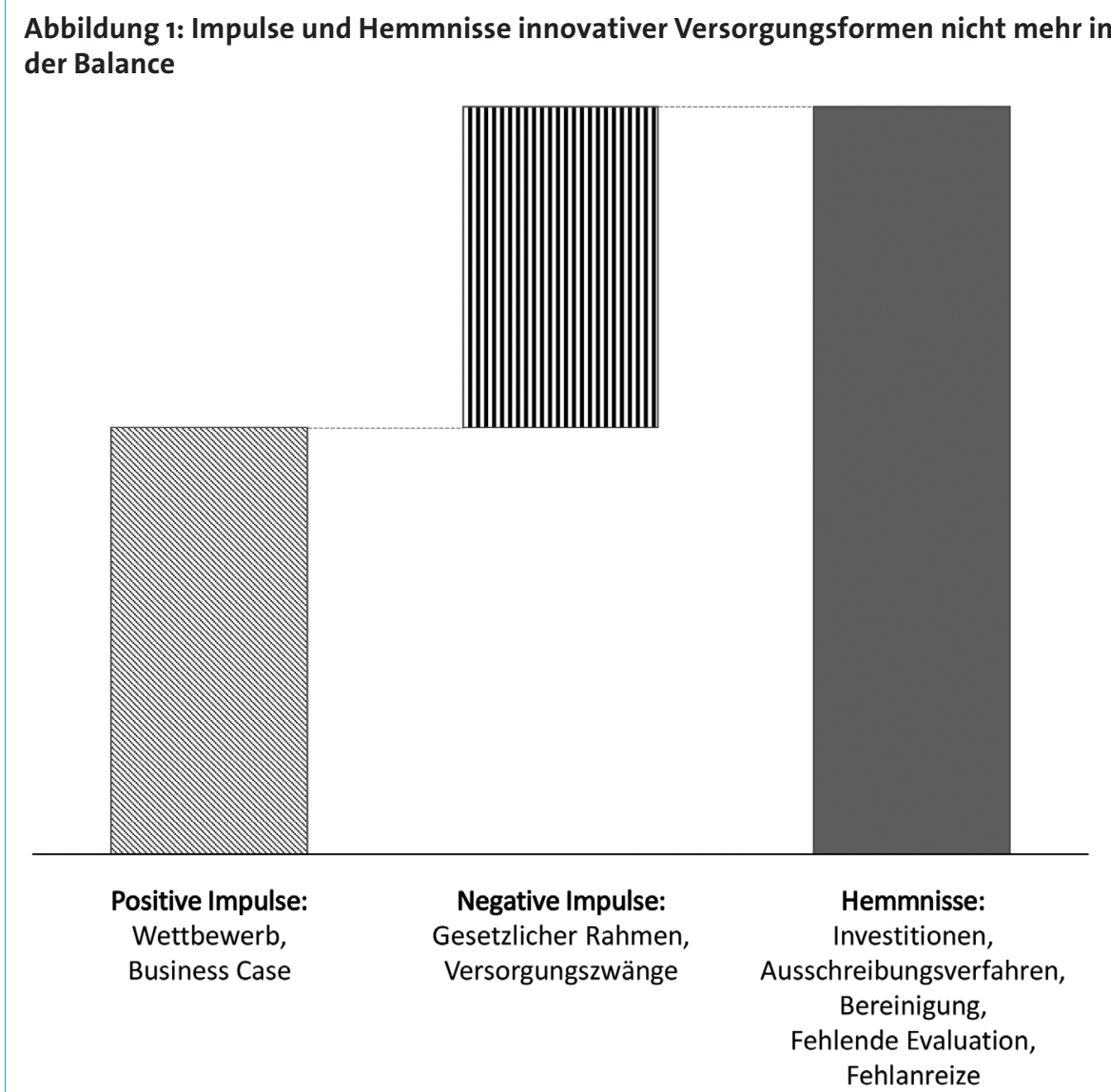

Quelle: Eigene Darstellung in Anlehnung an Amelung/Wolf 2013a, S. 98.

Unter den bisherigen Finanzierungsbedingungen mit Gesundheitsfonds, Morbi-RSA und pauschalen Zusatzbeiträgen haben die Krankenkassen gerade bei den Prozessinnovationen, die sich nicht nur auf die medizinischen Prozesse, sondern auch auf die Organisationsprozesse beziehen, starke Zurückhaltung gezeigt (vgl. Abbildung 1 und Neubauer 2012, S.3).

Impulse und Hemmnisse innovativer Versorgungsformen scheinen sich nicht mehr in der Balance zu befinden. Das Pendel scheint in Richtung der negativen Impulse bzw. der Hemmnisse umzuschlagen. Auch die zu geringe Ergebnisorientierung der verschiedenen Vergütungsverfahren kann als ein solcher Prozessmangel angesehen werden. Der grundsätzlich von allen beteiligten gew ünschte Wettbewerb ist - vor allem zwischen den Leistungserbringern - trotz diverser Reformen nicht sehr ausgeprägt. Inzwischen gibt es zwar die Möglichkeit, individuelle Verträge zwischen einzelnen Anbietern und den Krankenkassen abzuschließen, dies wird aber kaum für eine echte sektorunabhängige Versorgung genutzt. Im gegenwärtigen GKV-System wird beharrlich an ineffizienten Strukturen festgehalten, trotz besserer Alternativen und Konzepte. Die Forschung verwendet dafür den Ausdruck der Pfadabhängigkeit: In der Vergangenheit getroffene Entscheidungen engen das Spektrum möglicher künftiger Entscheidungen stark ein, das Verlassen des eingeschlagenen Weges ist nur unter Inkaufnahme hoher Kosten möglich und erklärt einen so genannten Lock-inEffekt. Mit Blick auf den Innovationsfonds bedarf es daher der Ausarbeitung konkreter Schritte und Förderprinzipien, damit der Fonds nicht, wie bereits befürchtet, floppt: „...zudem klappt die partnerschaftliche Entwicklung innovativer Versorgungsmodelle nicht per Ausschreibung" (Jacobs 2014, S.9).

Die ökonomische Analyse hat eine stärkere Nachfrageorientierung bei Entscheidungen über den Innovationsfonds ergeben. Mit Blick auf den Innovationsfonds sollten die Festlegungen und Entscheidungen des G-BA daher stärker nachfragebezogen ausfallen und den Versicherten/ Patienten stärker als bisher einbeziehen bzw. berücksichtigen. Damit läge man auch in dem internationalen Trend einer stärkeren Nutzerorientierung (vgl. SVR-G 2003, Kolstad/Chernew 2008).
Der Sachverständigenrat zur Begutachtung der Entwicklung im Gesundheitswesen hat diese Aspekte sehr ausführlich und sehr konkret in seinem Sondergutachten 2012 beleuchtet (vgl. SVR-G 2012). Von den vier thematisierten Wettbewerbsfeldern, die jeweils verschiedene Vertragsebenen, -partner sowie -inhalte aufweisen, kommt mit Blick auf den Innovationsfonds insbesondere dem vierten Wettbewerbsfeld eine zentrale Bedeutung zu. Dieses Wettbewerbsfeld bildet das selektivvertragliche System, in dem die einzelnen Krankenkassen mit den Leistungserbringern dezentrale Verträge über Mengen, Preise und Qualitäten der Leistungen schließen können. Der Innovationsfonds sollte daher die Förderung auf der Grundlage der besonderen Versorgungsformen vornehmen, d.h. eine versorgungsvertragliche Basis besitzen. Die konkrete Umsetzung des Innovationsfonds entscheidet, ob und wie effizient, effektiv und nachhaltig der Innovationsfonds wird oder ob er sich doch wieder nur in der Befriedigung sektoraler Ansprüche verliert. Das selektivvertragliche System steht allerdings nicht isoliert neben dem kollektivvertraglichen System, das nach wie vor eine Dauerbaustelle der Gesundheitspolitik ist. Hier besteht zwar kein Wettbewerb zwischen den Krankenkassen, die ihre Verträge weitgehend gemeinsam und einheitlich schließen, gleichwohl stehen auch hier die Leistungserbringer in Konkurrenz um die Nachfrage der Versicherten und der Patienten. Mit Blick auf die beiden Wettbewerbsfelder kollektiv und selektiv geht es damit auch um Finanzvolumina für Versorgungsfelder, die über die Beträge hinausgehen, die grundsätzlich aus einer Bereinigung der Regelversorgung zur Verfügung stehen.

Für einen funktionsfähigen Innovationsfonds muss der Gesetzgeber einen Freiraum insbesondere für Prozessinnovationen schaffen, weniger für Produktinnovationen, die beispielsweise in der Medizintechnik oder in der pharmazeutischen Industrie über zeitlich befristete Monopole (Patentschutz) grundsätzlich refinanziert werden können und nicht unbedingt einer Anschubfinanzierung bedürfen. Solche Innovationen finden bereits auf einer Vielzahl von Feldern im Gesundheitswesen statt, beispielsweise bei Maßnahmen zur Qualitätssicherung, bei Entwicklung und Aufbau von Kommunikationsnetzwerken, bei sektorübergreifenden Vergütungsmodellen oder auch bei medizinischen und klinischen Behandlungspfaden, welche an- 
streben, die starren Sektorengrenzen hinter sich zu lassen. Zur Entscheidungsfindung können hier implizite oder explizite Kriterienlisten dienen. Die Grundlage für förderungsfähige Innovationen kann $\mathbb{1 4 0 b}$ SGB V bilden, der die Vertragsformen zur Integrierten Versorgung benennt. Da die Nummern 8 und 9 des $\$ 140$ b SGB V die pharmazeutischen Unternehmen und die Hersteller von Medizinprodukten benennen, würden diese nach dem oben Gesagten nicht zu den antragsberechtigten Partnern für den Innovationsfonds zählen.

Mit Blick auf die zu fördernden Prozessinnovationen lautet das Ziel, Qualität und Wirtschaftlichkeit im Interesse der Patienten und Versicherten zu erhöhen. Denn divergierende Regulierungen der Sektoren behindern bislang eine Optimierung der verzahnten Behandlungsabläufe und schöpfen die jeweils zugewiesenen Ressourcen letztlich nur rein sektorspezifisch aus. Der Innovationsfonds sollte daher den Modellcharakter einer zukunftsfähigen, qualitätsgesicherten und patientenorientierten Konzeption aufweisen, die einen Anschub für die angestrebte sektorunabhängige Versorgung bildet. Dazu zählt auch, dass innovative Prozesse neu bzw. anders als bisher gedacht werden und Bekanntes - Dienstleistungen, Produkte, Strukturen - in Versorgungsketten neu zusammengeführt wird.

Um Wettbewerbsverzerrungen durch Zuweisungen an einige wenige Projekte zu verhindern, sollten Höchstgrenzen definiert werden und auch nur eine Teilfinanzierung vorgenommen werden (vgl. Amelung/Wolf 2013, S.51). Weiterhin sollten die Zuweisungen auf eine Förderperiode von 4 Jahren begrenzt bleiben, mit einer vollständigen externen Evaluation über die gesamte Laufzeit. Die Evaluation sollte dabei die Bedeutung der Begleitforschung betonen und ihre Ergebnisse und Erkenntnisse sollten für Dritte nutzbar gemacht werden. Es geht an dieser Stelle nicht um die Substitution der Evaluation in der Grundlagenforschung, sondern um eine versorgungsprozessbezogene Evaluation, die zu unmittelbaren Lerneffekten beiträgt. Diese Sichtweise wird auch geprägt von der bisherigen unzureichenden gesetzlichen Evaluation der strukturierten Behandlungsprogramme. Eine allgemeine Evaluation bzw. Grundlagenforschung sollte im Unterschied zur versorgungsprozessbezogenen Evaluation zudem stets steuerfinanziert sein, da sie der Verfolgung gesellschaftlicher Interessen dient.

\subsection{Governance-Aspekte}

Unter Governance-Aspekten des Innovationsfonds wird im Folgenden verstanden, wie die Steuerungs- und Entscheidungsstrukturen des Innovationsfonds ausgestaltet werden. Governance bezieht sich auf die Strukturen sowie die institutionellen respektive prozessualen Elemente der Innovationsförderung, wodurch das Fondsmanagement unterstützt und verbessert werden soll. In diesem Zusammenhang spielen die so genannten 4 W eine Rolle:

- Was soll gefördert werden, in welchem Umfang und für wie lange?

- Wer darf beantragen und wer entscheidet über die Mittelvergabe?

- Welche Rahmenbedingungen sind für ein funktionierendes Konzept erforderlich?

- Wie erreicht man die erwünschte Zielgenauigkeit bei der Innovationsförderung?

Das erste W „Was soll gefördert werden, in welchem Umfang und für wie lange? “ wurde bereits im Abschnitt 3.1 Förderaspekte diskutiert. Im Wesentlichen geht es um die Förderung sektorunabhängiger Prozessinnovationen, die im Rahmen einer Teilfinanzierung für maximal 4 Jahre gefördert und parallel dazu evaluiert werden. Die Basis bildet hierfür $\int 140$ b SGB V. Weitere Erfolgsanreize über Rückerstattungsmodelle sind ebenfalls möglich und diskussionswürdig ${ }^{3}$ (vgl. KBV 2014).

Antragsberechtigt können alle Partner der Integrierten Versorgung nach $\mathbb{} 140$ b SGB V sein, ohne Medizintechnik und ohne pharmazeutische Hersteller. Für eine Förderung durch den Innovationsfonds kommen sie nicht in Frage. Zum einen geht es bei diesen beiden Stakeholdern überwiegend um neue Produkte und nicht um Prozesse, zum anderen wird das Geld von den Krankenkassen per Umlage zur Verfügung gestellt und kann wohl kaum zur Finanzierung neuer Medizintechnik bzw. neuer Arzneimittel privatwirtschaftlicher Unternehmen herangezogen werden. Sicherlich erreicht man das größte Innovationspotenzial, wenn vielen Akteuren ein Antragsrecht zugebilligt wird. Wer sich in der Lage sieht, Versorgungsstrukturen und -prozesse zu verbessern, ist dazu aufgerufen. Um innovative Versorgungskonzepte insgesamt gezielt zu fördern, schlägt der SVR-G vor, klare Auswahlkriterien zu benennen u.a. eine Beschränkung auf sektorübergreifende Projekte, eine verpflichtende Evaluation sowie eine Priorisierung von populationsbezogenen (indikationsübergreifenden) Versorgungskonzepten (vgl. SVR-G 2012, Zi. 398).

Über die Mittelvergabe wird der G-BA als legitimiertes Gremium der Selbstverwaltung entscheiden. Gegenüber dem G-BA gibt es im Kontext des Innovationsfonds allerdings berechtigte Vorbehalte, denn von seiner Struktur her ist er der Inbegriff des sektoralen Gesundheitswesens. Fast alle Entscheidungen werden hier aufgrund der Stimmenverteilung auf die jeweiligen Bänke getroffen, und es erscheint eine contradictio in adiecto, sich vom G-BA Impulse für die anvisierte sektorübergreifende Ausrichtung des Innovationsfonds zu erhoffen. Mit Blick auf den Innovationsfonds sollte daher über die Stimmenverteilung im G-BA nachgedacht werden. Unter GovernanceAspekten erscheint es sinnvoll, die wissenschaftliche Bewertung der Anträge von der Entscheidung über die Förderung zu trennen. Der G-BA entscheidet zunächst über die Förderkriterien. Ein entsprechender Unterausschuss, Beirat, unabhängiges Institut oder eine Stiftung könnten für die wissenschaftliche Bewertung der Förderanträge zuständig sein. Vor dem Hintergrund der anvisierten nachfragebezogenen Entscheidung des G-BA wäre es auch interessant, den Krankenkassen in Kombination mit den Versicherten/Patienten die Erstellung der Förderliste im Verbund mit unabhängigen Gutachtern zu übertragen. In diesem Kontext wäre auch über eine zeitliche Priorisierung bzw. eine Quotierung der Mittel nachzudenken, falls die Fondsmittel nicht zur Finanzierung aller positiv geprüften Projekte ausreichen.

Unter Governance-Aspekten ist auch noch die Bestimmtheit der Förderverträge von erheblicher Relevanz. Die zentralen Punkte hierbei sind:

- Einheitliche Aufsicht

- Festlegung der zu erfassenden Dimensionen der Förderung

3 Ist ein Markteintritt leicht möglich, d. h. liegen nur wenige Marktschranken vor, so spricht man von einem bestreitbaren Markt. In diesem Kontext könnten das beispielsweise erhöhte Förderquoten für positiv evaluierte Projekte sein. 
- Realer Versorgungsbezug der Anträge

- Einhaltung von Standards

- Evaluation als integraler Bestandteil der Verträge.

\subsection{Implikationen für eine sektorunabhängige Versorgung}

Die strukturellen Defizite des deutschen Gesundheitswesens sind seit langem bekannt, dennoch können sich ineffiziente Strukturen beharrlich halten (vgl. Amelung/Wolf 2013a, S.97). Vorgegebene Grenzen, Fehlanreize und verkrustete Entscheidungsstrukturen stehen der Weiterentwicklung einer Innovationskultur entgegen. Der Innovationsfonds wird aber nur dann die gesetzten Ziele erreichen können, wenn die Spielregeln der Integrierten Versorgung und des Innovationsfonds zueinander passen. Es gilt daher, die Zielgenauigkeit der geförderten Projekte zu überprüfen und gegebenenfalls nachzusteuern zu können.

Interessant ist auch die Frage, ob es sich um einen zeitlich befristeten Finanzierungsschub durch den Innovationsfonds handelt? Geht man davon aus, dass zunächst lediglich ein Anstoß gegeben werden soll und berücksichtigt man die künftige Finanzsituation der Krankenkassen, wird man kaum von einer dauerhaften Belastung der Krankenkassen durch die Finanzierung des Innovationsfonds ausgehen können. Nach 4 Jahren sollte sich auch eine gewisse Eigendynamik entwickelt haben. Durch die Wiedererlangung eines Teils der Beitragssatzautonomie der Krankenkassen (zumindest mit Blick auf den kassenindividuellen prozentualen Zusatzbeitrag oberhalb von 14,6\% durch das GKV-Finanzstrukturund Qualitäts-WeiterentwicklungsgesetzGKV-FQWG) können die Krankenkassen ab 2015 wieder verstärkt in Innovationen investieren, ohne die massiven Wettbewerbsnachteile zu befürchten, wie sie bei dem pauschalen Zusatzbeitrag beklagt wurden. Damit käme dem Innovationsfonds auch eine Brückenfunktion mit Blick auf die neue Finanzarchitektur in der GKV zu (vgl. Neubauer 2012, S.4).

Viele Vorschläge zur Weiterentwicklung der sektorunabhängigen Versorgung in Deutschland konzentrieren sich auf die hohen Transaktionskosten eines IV-Vertrages im bestehenden System, insbesondere auf die Aspekte der Budgetbereinigung sowie auf das Vertragsmanagement (vgl. Braun/Greiner 2008,
S. 50). Der Innovationsfonds könnte hier eine entsprechende Ausgestaltung vorausgesetzt eine Brücke schlagen in Richtung auf eine „echte“ sektorunabhängige Versorgung. Dabei könnte er auch die Rolle nachfrageseitiger Entscheidungen und hier die des häufig vergessenen Players „Versicherter/Patient“ stärken. Eine wettbewerblich ausgerichtete GKV sollte sich auch stärker an den Präferenzen der Versicherten/Patienten orientieren. Falls die konkrete Ausgestaltung des Innovationsfonds in diese Richtung geht und nicht nur „Neues ausprobieren“ hilft, könnte der Fonds auch den Wettbewerb zwischen den Versorgungssystemen befeuern. Die geschilderte Orientierung an der Nachfrageseite könnte die sektorunabhängige Versorgung stärker als bisher

\section{Literatur}

Amelung, V. und Wolf, S. (2013a): Innovationsförderung in der GKV. In: Gesundheits- und Sozialpolitik, 2-3(2013):97-103.

Amelung, V. und Wolf, S: (2013b): Innovationsfonds. Von der guten Idee zur richtigen Umsetzung. In: Gesundheits- und Sozialpolitik, 5(2013):49-52.

Arrow, K. J. (1963): Uncertainty and the Welfare Economics of Medical Care. In: American Economic Review 53(1963): 941-73.

Braun, S. und Greiner, W. (2008): Die Übernahme von Budgetverantwortung durch Anbieter der Integrierten Versorgung. In: Gesundheitsund Sozialpolitik, 5(2008):49-52

Cassel, D. et al. (Hrsg.) (2014): Solidarische Wettbewerbsordnung. Grundprinzipien, Reformbaustellen und Perspektiven. Genese, Umsetzung und Perspektiven einer Konzeption zur wettbewerblichen Gestaltung der gesetzlichen Krankenversicherung, medhochzwei, Heidelberg.

Ehlert, A. und Wein, T. (2014): Integrierte Versorgung - Wettbewerbspotenzial in der Gesundheitsversorgung heben!

In: Wirtschaftsdienst 3(2014):194-202

Enthoven, A. (1978): , Consumer-ChoiceHealth-Plan: A national health insurance proposal on regulated competition in the private sector", In: New England Journal of Medicine 298:709-720.

Enthoven, A.C. (1993b): The History and Principles of Managed Competition. In: Health Affairs, 12(SI):24-48.

v. Hayek, F. A. (1972): Die Theorie komplexer Phänomene; Mohr Siebeck, Tübingen.

Jacobs, K. (2014): Neuer Schub für Ideen: In: Ausgabe 1(2014), 17. Jahrgang:9.

Kantzenbach, E. (1967): Die Funktionsfähigkeit des Wettbewerbs, 2. Auflage, Vandenhoeck \& Ruprecht, Göttingen.

KBV- Kassenärztliche Bundesvereinigung

(2014): Positionierung der Kassenärztlichen in Konkurrenz zur Regelversorgung stellen (vgl. Ehlert und Wein 2014, S.201).

Ein Vergleich mit entsprechenden Entwicklungen in der Schweiz oder den Niederlanden zeigt, dass in Deutschland der Wettbewerb zwischen den Versorgungssystemen durch Eintrittsbarrieren und Regulierungen bislang nur schwach ausgeprägt ist. Die einzelnen Akteure versuchen, durch Rent-Seeking-Aktivitäten Vorteile aus dem Gesundheitsfonds zu erzielen und verfolgen dabei im Wesentlichen ihre eigenen Partikularinteressen, wodurch ein zu geringes Angebot an sektorunabhängigen Versorgungsformen bei zu hohen Preisen oder zu geringen Qualitäten entsteht (Wille et al. 2007, S. 34). Würde der Innovationsfonds in diese Richtung ausgestaltet, hätte er seinen Namen verdient. CDU, CSU und SPD zum Abschnitt Gesundheit und Pflege (18. Legislaturperiode), Berlin.

Kolstad, J., und Chernew, M. (2008): Quality and Consumer Decision Making in the Market for Health Insurance and Health Care Services. In: Medical Care Research and Review, 66(1):28S-52S.

Neubauer, G. (2012): Errichtung eines Innovationsfonds für die GKV. In: Chefarzt aktuell, 5(2012):1-4

Rebscher, H. (1993): Wettbewerb und Solidarität - Skizze einer solidarischen Wettbewerbsordnung für die GKV. In: Die Ersatzkasse. 1993 S. $4 \mathrm{ff}$.

SVR-G - Sachverständigenrat zur Begutachtung der Entwicklung im Gesundheitswesen (2003): Finanzierung, Nutzerorientierung und Qualität, Gutachten 2003, Band I und II, Nomos, Baden-Baden.

SVR-G - Sachverständigenrat zur Begutachtung der Entwicklung im Gesundheitswesen (2012): Wettbewerb an der Schnittstelle zwischen ambulanter und stationärer Gesund heitsversorgung, Sondergutachten 2012, Hans Huber, Bern

Wasem, J. (2011): Wie muss Wettbewerb weiter ausgestaltet werden?, Vortrag, AOK im Dialog „Zukunft der GKV: Solidarität bewahren-durch mehr Wettbewerb",

Berlin, 15. November 2011

Wille, E. et al. (2007): Die Weiterentwicklung des Krankenversicherungsmarktes: Wettbewerb und Risikostrukturausgleich. In: Wille, E. et al. (Hrsg.): Wettbewerb und Risikostrukturausgleich im internationalen Vergleich, Nomos, Baden-Baden: 15-68.

Wille, E. (2009): Die Empfehlungen des Sachverständigenrates zur integrierten Versorgung, in: Wille, E. und Knabner, K. (Hrsg.), Die besonderen Versorgungsformen: Herausforderungen für Krankenkassen und Leistungserbringer, Frankfurt: 91-123. 